\title{
Ibuprofen-loaded fibrous patches-taming inhibition at the spinal cord injury site
}

\section{Liliana R. Pires 1,5 , Cátia D. F. Lopes ${ }^{1,2}$, Daniela Salvador', Daniela N. Rocha1, Ana Paula Pêgo',2,3,4}

1.INEB - Instituto de Engenharia BiomédicaUniversidade do PortoPortoPortugal 2.i3S - Instituto de Investigação e Inovação em SaúdeUniversidade do PortoPortoPortugal 3.Instituto de Ciências Biomédicas Abel SalazarUniversidade do Porto (ICBAS) PortoPortugal 4.Faculdade de Engenharia da Universidade do Porto (FEUP) PortoPortugal 5.INL- International Iberian Nanotechnology LaboratoryBragaPortugal Corresponding Author:E-mail: apego@ineb.up.pt

Originally published in: Journal of Materials Science: Materials in Medicine (2017) 28: 157. https://doi.org/10.1007/s10856-017-5967-7

"This is a post-peer-review, pre-copyedit version of an article published in Journal of Materials Science: Materials in Medicine. The final authenticated version is available online at: https://doi.org/10.1007/s10856-017-5967-7."

\begin{abstract}
It is now widely accepted that a therapeutic strategy for spinal cord injury ( $\mathrm{SCl}$ ) demands a multitarget approach. Here we propose the use of an easily implantable bilayer polymeric patch based on poly(trimethylene carbonate-co- $\varepsilon$-caprolactone) $(\mathrm{P}(\mathrm{TMC}-\mathrm{CL})$ ) that combines physical guidance cues provided by electrospun aligned fibres and the delivery of ibuprofen, as a mean to reduce the inhibitory environment at the lesion site by taming RhoA activation. Bilayer patches comprised a solvent cast film onto which electrospun aligned fibres have been deposited. Both layers were loaded with ibuprofen. In vitro release $\left(37^{\circ} \mathrm{C}\right.$, in phosphate buffered saline) of the drug from the loaded scaffolds under sink condition was found to occur in the first $24 \mathrm{~h}$. The released ibuprofen was shown to retain its bioactivity, as indicated by the reduction of RhoA activation when the neuronal-like cell line ND7/23 was challenged with lysophosphatidic acid. Ibuprofen-loaded $P(T M C-C L)$ bilayer scaffolds were successfully implanted in vivo in a dorsal hemisection rat $\mathrm{SCl}$ model mediating the reduction of RhoA activation after 5 days of implantation in comparison to plain $\mathrm{P}(T M C-C L)$ scaffolds. Immunohistochemical analysis of the tissue shows $\beta$ III tubulin positive cells close to the ibuprofenloaded patches further supporting the use of this strategy in the context of regeneration after a lesion in the spinal cord.
\end{abstract}

\section{INTRODUCTION}

\subsection{Ana Paula Pêgo-Career goals}


I see myself as an Engineer of the Regeneration. Solving problems is my driving force. Nothing motivates me more! And promoting nervous tissue regeneration has been an unbeatable challenge. Our knowledge of the processes that rule nervous tissue regeneration expanded a lot in recent years. But there is still a long way towards translating that knowledge into effective therapies. Biomaterials have been my tool. And there is so much we still do not know about biomaterials to interface with the nervous system!

I believe that having found a topic that fascinates and excites me was paramount. But it was equally important to have had mentors that inspired me, guided me in my training without restricting my creativity and ideas (they trusted me which gave me confidence); that empowered me, for good and for bad, to make my own decisions; that taught me the importance of sharing our knowledge with peers and the society, and building a strong network of collaborators.

Starting my own group has been an important step. But keeping it bustling continues to be a challenge. One needs to pursue one's objectives, while finding the right team members and funding. I believe that building a team with complementary skills has been very important for the work we have been performing. In terms of funding I believe that I started writing better proposals when I started reviewing grants myself. So, don't say no when invited to act has a reviewer!

Reflecting on my career path this is the legacy that I also try to pass on to the younger generations that I have the privilege to work with. A group of motivated, passionate and bright researchers that also want to follow their dreams!

\subsection{Engineering biomaterials to promote regeneration after spinal cord injury}

Injury to the spinal cord is marked by the disruption of ascending and descending axonal pathways, interrupting the communication between the brain and other parts of the body. In the adult central nervous system (CNS) axonal regrowth after injury, necessary to restore connectivity, is prevented by the lack of physical support and by the presence of inhibitory molecules in the extracellular milieu (see $[\underline{1}, \underline{2}, 3]$ for a review). The Ras homolog gene family member A - RhoA - is considered a key convergence effector molecule for several of these inhibitory pathways. It has been demonstrated that RhoA antagonists can overcome axonal growth inhibition, promoting axonal regeneration and functional recovery after injury in the CNS $[4,5, \underline{6}]$. Consequently, taming RhoA activation has been pointed out as a promising therapeutic approach for spinal cord injury $(\mathrm{SCl})[4, \mathrm{Z}]$.

In 2003, it was reported for the first time that non-steroidal anti-inflammatory drugs (NSAIDs), such as ibuprofen, can inhibit RhoA signalling [8]. Ibuprofen has been generally used for pain relief, lowering fever and reducing inflammation, based on its inhibitory action on cyclooxygenases [2, 10]. In the specific context of SCI treatment, different research groups demonstrated that, by effectively blocking Rho-activation, the administration of ibuprofen promotes functional recovery $[\underline{11}, 12,13]$ and corticospinal axonal regeneration [11]. The administration of ibuprofen has also been associated with reduction of neuropathic pain [1ㄷ] and increase on oligodendrocyte survival and axonal myelination [15], further supporting the use of this drug in the context of $\mathrm{SCl}$ recovery. The most common risks associated to the long-term use of NSAIDs are the occurrence of gastrointestinal ulcers and hemorrhage and, more rarely, acute renal failure during treatment [16]. This is considered an acceptable risk profile, as in the setting of acute care of $\mathrm{SCl}$ patients, risk factors can be controlled by 
close in-hospital monitoring [16]. Based on the above, assuming therapeutic equivalence, ibuprofen holds great potential to become a new therapeutic tool in $\mathrm{SCl}$ as a small-molecule-mediated Rhoinhibitor, with clear advantages in economic terms in comparison to other recently developed approaches [16].

There is increasing evidence that the multifaceted inhibitory nature of $\mathrm{SCl}$ demands a multi-target combinatorial therapeutic approach $[\underline{1}, \underline{17}]$. The work herein presented aimed at designing a scaffold that combines physical guidance for axonal growth and the delivery of ibuprofen. The ultimate goal is to create a device that can be easily implanted, providing multiple signals that contribute to convert the lesion site into a more permissive environment for regeneration. Poly(trimethylene carbonateco- $\varepsilon$-caprolatone) $[\mathrm{P}(\mathrm{TMC}-\mathrm{CL})]$ was selected as the starting material for the development of a bilayer patch in which the layer that contacts the lesion area is based on longitudinally aligned fibres. In a $\mathrm{SCI}$ scenario, the use of aligned fibrous structures is of particular interest, as these can provide physical guidance cues for neurons, allowing cellular processes to orient in the direction of the fibres $[\underline{18}, \underline{19}]$ and, ultimately, lead to improved regeneration [20]. P(TMC-CL) is a synthetic copolymer previously applied in the preparation of nerve conduits [21]. It has been demonstrated that $P(T M C-C L)$ can support peripheral nerve regeneration in vivo [22] and, in the context of the CNS, P(TMC-CL) showed to stimulate neuronal polarization and axonal elongation, favouring neurite outgrowth when in an inhibitory environment [23]. Our group has previously described the preparation of randomly oriented $\mathrm{P}(\mathrm{TMC}-\mathrm{CL})$ fibres by electrospinning $[24]$ and showed in vitro that fibrous or flat $\mathrm{P}(\mathrm{TMC}-\mathrm{CL})$ films provide cues that may guide microglia, the resident immune cells of the CNS, towards a proregenerative profile [25]. Moreover, it was demonstrated that these homogenously drug-loaded fibrous structures mediate the release of ibuprofen over $24 \mathrm{~h}$, yielding fibres with anti-inflammatory properties [24]. Here, the preparation of anisotropic ibuprofen-loaded P(TMC-CL) scaffolds is described and their performance on the inhibition of RhoA pathway both in vitro and in vivo, is investigated.

\section{Materials and methods}

\subsection{Preparation of P(TMC-CL) patches}

\subsubsection{Copolymer synthesis and characterization}

Bilayer scaffolds were prepared using a statistical P(TMC-CL) copolymer. The copolymer was synthesized by ring-opening polymerization and subsequently purified as previously described [26]. In brief, $\varepsilon$-caprolactone (CL) (Merck, Germany) was dried overnight using calcium hydride (SigmaAldrich Química, Portugal) and distilled before the polymerization with trimethylene carbonate (TMC, used as received from Boehringer Ingelheim, Germany). Polymerization was carried out in evacuated and sealed glass ampoules using stannous octoate (Sigma-Aldrich Química, Portugal) as catalyst $\left(2 \times 10^{-4} \mathrm{~mol}\right.$ per mol of monomer). After 3 days of reaction at $130^{\circ} \mathrm{C}$ the polymer obtained was purified by dissolution in chloroform (BDH-Prolabo, Portugal) and subsequent precipitation into a tenfold volume of ethanol ( $96 \%(\mathrm{v} / \mathrm{v}), \mathrm{AGA}$, Portugal). The chemical composition of the prepared copolymer was assessed by ${ }^{1} \mathrm{H}$ nuclear magnetic resonance (NMR) and found to contain $11 \% \mathrm{~mol}$ of TMC, being in accordance to the monomer ratio charged ( $10 \%$ mol TMC). The average number molecular weight and polydispersity index of the purified polymer were assessed by size exclusion chromatography [24] and determined to be $8.2 \times 10^{4}$ and 1.61, respectively. 


\subsubsection{Bilayer patch fabrication}

Bilayer patches (see Fig. 4a) comprise an outer layer based on a solvent cast P(TMC-CL) film, and an inner layer made of preferentially aligned electrospun $\mathrm{P}(\mathrm{TMC}-\mathrm{CL})$ fibres. To prepare solvent cast films, a $6 \%$ (w/v) P(TMC-CL) solution in dichloromethane (DCM; Merck, Germany) was casted onto a glass mould. The solvent was left to evaporate at room temperature $\left(20-25^{\circ} \mathrm{C}\right)$ overnight under a DCM saturated atmosphere and, subsequently, the films were vacuum dried for $24 \mathrm{~h}$ (vacuum oven, Raypa, Spain). $\mathrm{P}(\mathrm{TMC}-\mathrm{CL}$ ) electrospun fibres were prepared as previously reported [24] . Briefly, $\mathrm{P}(\mathrm{TMC}-\mathrm{CL})$ was dissolved in a mixture of $\mathrm{DCM}$ and $\mathrm{N}, \mathrm{N}$-dimethylformamide (DMF, Merck) at a volume ratio of 3:1. The solution was dispensed at $1 \mathrm{ml}^{-h^{-1}}$ through a blunt needle (inner diameter $0.8 \mathrm{~mm}$ ) placed at $13 \mathrm{~cm}$ from the collector. An $18 \mathrm{kV}$ electric field was applied (Gamma High Voltage source; $F L, U S A)$ and the fibres were collected during 2.5 h onto $P(T M C-C L)$ solvent cast films. A cylindrical drum ( $10 \times 15 \mathrm{~cm}$, Yflow, Spain) rotating at $3000 \mathrm{rpm}$ was used as fibre collector. $6 \times 5 \mathrm{~mm}$ bilayer scaffolds were punched out and packed under vacuum after an argon purge. Samples were sterilized by gamma irradiation ( $25 \mathrm{kGy},{ }^{60} \mathrm{Co}$ source) prior further use.

For the preparation of ibuprofen-loaded patches, the drug was added to the polymer solution and slowly stirred for $5 \mathrm{~h}$ before casting or electrospinning. Both layers of the scaffold were loaded with $5 \%$ (w/w of polymer) of ibuprofen (pharmaceutical grade, kind offer of Sérgio Simões - Bluepharma, Coimbra, Portugal).

\subsubsection{Patch characterization}

The patches were observed by scanning electron microscopy (SEM) using a FEl Quanta 400FEG microscope (FEl, the Netherlands) after being sputter-coated with gold-palladium for $90 \mathrm{sec}$ (SPI Supplies, PA, USA). Fibre diameter and alignment were quantified from SEM micrographs using image analysis software (Image J, version 1.39, NIH, MD, USA). The fibre mean diameter was calculated by analysing micrographs from 3 independently obtained fibrous meshes. For each sample (fibre mesh), 100 measurements were conducted. To calculate fibre alignment, the preferential direction of the alignment was defined for each image and subsequently the angle formed between the fibres and this axis measured. Results are presented as percentage relative to the total number of fibres measured from 3 independent samples.

\subsection{Biofunctionality assessment of ibuprofen-loaded P(TMC-CL) patches in vitro}

\subsubsection{Drug release quantification}

$\mathrm{P}(\mathrm{TMC}-\mathrm{CL})$ patches were incubated $\left(37^{\circ} \mathrm{C}, 120 \mathrm{rpm}\right)$ in phosphate buffered saline (PBS) at the final concentration of $5 \mathrm{mg} \mathrm{ml}^{-1}$ (mass of scaffold/volume of PBS). At defined time points $(0.5,1,2,4,6,8$, 24 and $48 \mathrm{~h}$ ) the medium was collected and refreshed. The amount of ibuprofen released was quantified by high-pressure liquid chromatography (HPLC) in a LaChrom Elite ${ }^{\circledast}$ system (Hitachi, Portugal) equipped with a variable wavelength diode array detector (L-2455). Separation was performed using a Lichrosorb RP-18 column $(5 \mu \mathrm{m}, 12.5 \times 0.4 \mathrm{~cm}$, Merck) and a mobile phase consisting on a mixture of acetonitrile and ortho-phosphoric acid $0.015 \mathrm{M}(45: 55$, ( $\mathrm{v} / \mathrm{v})$, all supplied by Merck). Flow rate was set to $2.5 \mathrm{ml} \mathrm{h}^{-1}$ and separation occurred at $40^{\circ} \mathrm{C}$, whereas detection was performed at $25^{\circ} \mathrm{C}$. Ibuprofen was detected at $230 \mathrm{~nm}$ after $10 \mu \mathrm{l}$ sample injection. Cumulative 
release was calculated relative to the theoretical maximum loading of $5 \%(\mathrm{w} / \mathrm{w}$ of polymer). The limit of quantitation of the applied HPLC setup was determined to be $0.001 \mathrm{mg} \mathrm{ml}^{-1}$.

\subsubsection{Cell culture}

$\mathrm{ND} 7 / 23$ cell line (mouse neuroblastoma ( $\left.\mathrm{N}_{18} \mathrm{tg} 2\right) \times$ rat dorsal root ganglion neuron hybrid) was obtained from ECACC (United Kingdom) and routinely cultured in Dulbecco's Modified Eagle Medium (DMEM) with Glutamax, supplemented with $10 \%(\mathrm{v} / \mathrm{v})$ of heat inactivated $\left(56^{\circ} \mathrm{C}\right.$, $\left.30 \mathrm{~min}\right)$ foetal bovine serum (FBS) and $1 \%$ penicillin/streptomycin (PS, 10,000 units $\mathrm{ml}^{-1}$ penicillin and $10,000 \mu \mathrm{g} \cdot \mathrm{ml}^{-1}$ streptomycin), all supplied by Gibco (Life technologies S.A., Spain). ND7/23 cells were sub-cultured $\left(2 \times 10^{4}\right.$ viable cells. $\left.\mathrm{cm}^{-2}\right)$ and incubated with DMEM supplemented with $0.5 \% \mathrm{FBS}, 1 \%$ PS, $200 \mathrm{nM}$ nerve growth factor (NGF, Calbiochem, Merck), and $1 \mathrm{mM}$ cyclic adenosine monophosphate (CAMP, Sigma-Aldrich) during 2 days to induce cell differentiation. The effect of ibuprofen on ND7/23 cell viability was assessed by means of a resazurin-based assay as previously described [24]. Different ibuprofen solutions in ethanol:water, (7:3) were applied, being that the final ibuprofen concentration in culture media ranged between o to $2000 \mu \mathrm{M}$.

\subsubsection{Inhibition of RhoA activation by ibuprofen-loaded P(TMC-CL) patches}

To investigate whether ibuprofen released from $\mathrm{P}(\mathrm{TMC}-\mathrm{CL}$ ) scaffolds could influence RhoA activation in ND7/23 cells, extracts from the ibuprofen-loaded patches (Ibuprofen patch) were prepared as follows. Scaffolds were incubated in DMEM at $37^{\circ} \mathrm{C}(8 \mathrm{o} \mathrm{rpm})$ during $72 \mathrm{~h}$. The volume of DMEM used was adjusted according to the weight of scaffolds in order to achieve, after the total release of ibuprofen, a theoretical drug concentration of $500 \mu \mathrm{M}$. Ibuprofen final concentration was selected based on a previous report showing ibuprofen-mediated inhibition of RhoA pathway in the PC12 cell line [11]. The obtained extracts were then tested on differentiated ND7/23 cells as represented in Fig. 1. In brief, after a starving period of $2 \mathrm{~h}$ in DMEM without serum, cell culture medium was replaced by the extracts obtained from the ibuprofen-loaded $\mathrm{P}(\mathrm{TMC}-\mathrm{CL}$ ) patches. Ibuprofen solution (100x in ethanol:water (7:3)) was added to the cell culture media to a final concentration of $500 \mu \mathrm{M}$ to serve as control (Ibuprofen soluble). After $30 \mathrm{~min}$, the cells were activated with $45 \mu \mathrm{M}$ of lysophosphatidic acid (LPA, Sigma-Aldrich) for $10 \mathrm{~min}$ [11]. Non-treated cells served as a negative control, and cells activated with LPA served as positive control for RhoA activation.

RhoA activation was assessed using Rhotekin RBD-coupled beads (Millipore, MA, USA) according to manufacturer instructions. Cell lysates were prepared and incubated with the beads, as previously described [11]. GTP-RhoA along with $25 \mu \mathrm{g}$ of total lysate were separated in a $12 \%(\mathrm{w} / \mathrm{v})$ SDSpolyacrilamide gel and detected by Western blot (anti-RhoA antibody, 1:500, sc-418; Santa Cruz Biotechnology, CA, USA; horseradish peroxidase-labelled sheep anti-mouse IgG, 1:3000, Amersham). The relative intensity of protein bands was analysed using Quantity One (version 4.6) program (Bio-Rad). Results are expressed as the ratio between the intensity of bands of the activated protein form of RhoA (RhoA-GTP) and the total RhoA. For each cell culture experiment the RhoA levels were normalized relative to RhoA basal activation.

\subsection{In vivo study}

\subsubsection{Animals and surgical procedures}


All animal experiments were carried out with the permission of the local animal ethical committee in accordance with the EU Directive (2010/63/EU) and Portuguese law (DL 113/2013). The experimental protocol (421/000/000/2014) was approved by the ethics committee of the Portuguese official authority on animal welfare and experimentation (Direção-Geral de Alimentação e Veterinária, DGAV). Female adult Wistar rats (10-13 weeks) were anesthetized with ketamine/medetomidine (100 $\mathrm{mg} \mathrm{kg}^{-1}$ ketamine; $1 \mathrm{mg} \mathrm{kg}^{-1}$ medetomidine). Laminectomies were performed at the 7 and 8 thoracic vertebrae and the spinal dura mater was incised to expose the spinal cord. Dorsal hemisection at the T8 level was performed using a micro-scissor. The scaffolds $(6 \times 5 \mathrm{~mm})$ were implanted underneath the dura, immediately after the lesion was carried out. The fibrous side of the patch was placed in contact with the spinal cord, ensuring that fibres were longitudinally aligned with the axonal pathways. The surgery site was closed in layers using absorbable suture (Surgicryl, Smi, Belgium). Atipamezole (1 $\mathrm{mg} \mathrm{kg}^{-1}$ ) was administrated to counteract anaesthesia. The rats were kept on heating pads $24 \mathrm{~h}$ after surgery. Abdominal massage was performed twice a day to help bladder evacuation. Buprenorphine ( $0.1 \mathrm{mg} \mathrm{kg}^{-1}$ ) was administrated twice a day during the first 3 days after surgery to manage pain. A total of 21 animals were used in this study distributed in four experimental groups as follows: (A) non-operated (no lesion) $(n=2)$, (B) non-treated $(n=7),(C) \mathrm{P}(\mathrm{TMC}-\mathrm{CL})$ patch $(n=6)$, (D) ibuprofen-loaded P(TMC-CL) patch $(n=6)$. Animals from group $\mathrm{B}, \mathrm{C}$ and $\mathrm{D}$ were subjected to dorsal hemisection as described above. Animals from group $B$ were subjected to no further treatment, and animals from group $C$ and $D$ were implanted with $P(T M C-C L)$ patches, plain or loaded with ibuprofen, respectively. 5 days after lesion, the animals were deeply anesthetized (ketamine/medetomidine) and perfused transcardially with PBS. The spinal cord was dissected and collected. Approximately $1 \mathrm{~cm}$ of tissue centred at the lesion site from 2 non-operated rats (group A) and 4 animals from groups $B, C$ and $D$ were snap frozen in dry ice and stored at $-80^{\circ} \mathrm{C}$ for posterior RhoA quantification. In order to allow immunohistochemical analysis, spinal cord tissue was fixed using $4 \%\left(\mathrm{w} / \mathrm{v}\right.$ ) paraformaldehyde at $4{ }^{\circ} \mathrm{C}$ (overnight). The samples ( 3 from group $\mathrm{B}$ and 2 from group $\mathrm{C}$ and $\mathrm{D})$ were then transferred to a $30 \%(\mathrm{w} / \mathrm{v})$ sucrose solution containing $0.1 \%(\mathrm{w} / \mathrm{v})$ sodium azide for cryoprotection and stored at $-20^{\circ} \mathrm{C}$ before cryostat sectioning.

\subsubsection{Assessment of RhoA activation levels}

Tissue lysates were prepared using MLB buffer containing the protease inhibitors cocktail additionally supplemented with phosphatase inhibitors (10 $\mathrm{mM}$ sodium fluoride, $1 \mathrm{mM}$ sodium orthovanadate, $2 \mathrm{mM}$ sodium pyrophosphate). Active RhoA (RhoA-GTP) in lysates was assessed using two methods: the RhoA activation assay kit (G-LISA; Cytoskeleton, Inc.) according to the supplier instructions, and the pull-down assay as described in the previous section. RhoA activation levels are presented as the ratio between the activated protein form of RhoA (RhoA-GTP) and the total RhoA detected in the tissue lysate determined by Western blot.

\subsubsection{Histology and immunohistochemistry}

Spinal cord tissue was embedded in optimum cutting medium (OCT, Thermo Scientific, Thermo Fisher Scientific, Portugal) and cut onto $16 \mu \mathrm{m}$ thick sections using a cryostat (Thermo Scientific). Sections were mounted onto glass coverslips (Superfrost, VWR) and stored at $-20^{\circ} \mathrm{C}$ till further use. Sections were stained using haematoxylin and eosin according to standard procedures. For immunohistochemistry, cryostat sections were firstly incubated with $0.1 \%(\mathrm{w} / \mathrm{v})$ sodium borohydride ( $5 \mathrm{~min}$ ) and ammonium chloride (50 mM, $15 \mathrm{~min}$ ). Afterwards, sections were incubated $1 \mathrm{~h}$ at $37^{\circ} \mathrm{C}$ with blocking buffer containing $5 \%(\mathrm{v} / \mathrm{v}$ ) normal donkey serum (Sigma-Aldrich) and $0.3 \%(\mathrm{v} / \mathrm{v}$ ) Triton 
X-100 in PBS. Sections for RhoA-GTP detection were pre-incubated with Rhotekin RBD-coupled beads (overnight, $4^{\circ} \mathrm{C}$ ). Subsequently, samples were incubated overnight $\left(4^{\circ} \mathrm{C}\right.$ ) with the primary antibodies. Antibodies used include: rabbit anti-glial fibrillary acidic protein (GFAP, Dako, 1:500), mouse anti- $\beta$ III-tubulin (Promega, 1:2000), mouse anti-CD163 (Santa Cruz Biotechnologies, 1:50), rabbit anti-IBA-1 (Wako, 1:500), and rabbit anti-GST (Z-5, Santa Cruz Biotechnologies, 1:50). Tissue sections were subsequently incubated $1 \mathrm{~h}$ at room temperature with the corresponding secondary antibodies (Alexa Fluor ${ }^{\oplus}$-conjugated anti-rabbit or anti-mouse, Invitrogen, 1:1000). Afterwards, Hoechst solution (Invitrogen, 1:30,000) was applied (15 min at room temperature) in order to label cell nuclei. Stained sections were coverslipped using Fluoromount (Sigma-Aldrich) and kept at $-20^{\circ} \mathrm{C}$ until analysis. The immunolabelled tissue sections were observed under an inverted fluorescence microscope (Axiovert 200, Zeiss, Germany). Semi-quantitative analysis of the captured images was performed by five independent observers. The lesion area was delimitated and cell infiltration was categorized according to the following scoring system: $(+/-)$ rare cells; $(+)$, few cells; $(++)$, some cells; $(+++)$, many cells.

\subsection{Statistical analysis}

Statistical analysis was performed using the software Graphpad Prism version 5.0 (GraphPad, CA, USA). Statistical differences were calculated using one-way ANOVA followed by the Dunnett's test for multiple comparisons. A $p<0.05$ was considered statistically significant.

\section{Results}

\subsection{P(TMC-CL) bilayer patch characterization}

Bilayer patches were prepared by combining solvent casting and electrospinning (Fig. 2). The outer layer, composed by a flat solvent cast film revealed a spherulitic morphology, typical of a semicrystalline material [27] (Fig. 2a, c, e, g). The inner layer composed by electrospun fibres showed a preferentially aligned fibrous structure (Fig. $\underline{2 b}, \mathrm{~d}, \mathrm{f}, \mathrm{h})$. The formation of bended fibres was denoted in the ibuprofen-loaded fibres, resulting from the increase on jet instability due to the presence of the drug in the electrospun solution (Fig. $\underline{2 f}$ and h $)$. Nonetheless, the fibre mean diameter and alignment were not significantly influenced by the presence of ibuprofen in the $P(T M C-C L$ ) solution (Fig. $2 i-k$ ). Fibre mean diameter was found to be 0.661 and $0.646 \mu \mathrm{m}$ for non-loaded and ibuprofen-loaded fibres, respectively (Fig. $\underline{2 k}$ ).

\subsection{Ibuprofen-loaded P(TMC-CL) bilayer patch-in vitro assessment of bioactivity}

The release of ibuprofen from the $\mathrm{P}(\mathrm{TMC}-\mathrm{CL})$ bilayer patches was evaluated in PBS at $37^{\circ} \mathrm{C}$ in sink conditions. Under these experimental settings, over $90 \%$ of the drug was released within the first $24 \mathrm{~h}$ of incubation in PBS (Fig. 3a). The scaffold morphology remained unchanged after drug release (SEM data, not shown).

The effect of increasing concentrations of ibuprofen on ND7/23 cell metabolic activity was assessed, being the latter herein applied as a measure of cellular viability. Ibuprofen in solution (ethanol:water mixture (7:3)) was added to the cell culture medium to a final concentration ranging from o to $2000 \mu \mathrm{M}$. The percentage of cell metabolic activity was calculated relative to the vehicle. Figure $3 \mathrm{~b}$ 
shows that when ND7/23 cells are incubated for $24 \mathrm{~h}$ with ibuprofen at a final concentration of $1000 \mu \mathrm{M}$ and above, the cell metabolic activity is significantly reduced in a concentration-dependent manner.

The effect of ibuprofen released from the prepared $P(T M C-C L)$ patches on RhoA-GTP levels of $\mathrm{ND} 7 / 23$ cells was assessed in order to find out whether the drug released from the scaffolds maintained its bioactivity. ND7/23 cells were incubated with extracts of ibuprofen-loaded patches obtained in cell culture medium and subsequently challenged with LPA. The results of the quantification of RhoA expression levels are presented in Fig. 3C. RhoA-GTP was not detected in nonstimulated ND7/23 cells (C-). Conversely, treatment with LPA triggered an increase in RhoA-GTP (LPA) that was prevented by pre-treatment with soluble ibuprofen (IBU-sol, $500 \mu \mathrm{M}$ ) or ibuprofen released from the prepared patches (IBU-patch, reduction of approximately 5 times).

\subsection{In vivo implantation of ibuprofen-loaded P(TMC-CL) patch}

The developed $\mathrm{P}(\mathrm{TMC}-\mathrm{CL})$ bilayer patches were tested in vivo in a rat dorsal hemisection spinal cord injury model. As depicted in Fig. $4 a$, the P(TMC-CL) patches (Fig. 3 b) were placed on top of the lesion, wrapping the spinal cord tissue, with the fibrous layer longitudinally aligned with the spinal cord. The patches are very flexible allowing these to bend around the spinal cord (Fig. $4 \mathrm{C}$ ), providing for the close contact with the spinal cord tissue. In this manner, the vertebrae created a natural holding system to prevent the dislocation of the patches during the implantation period. Overall, the implantation of ibuprofen-loaded $\mathrm{P}(\mathrm{TMC}-\mathrm{CL})$ patches, plain or loaded with ibuprofen, did not compromise animal survival after $\mathrm{SCl} .5$ days after implantation, the retrieved scaffolds were found to be covered by fibrous tissue, and no macroscopic signs of scaffold degradation were observed (Fig. 4d).

The effect of the ibuprofen released from the $P(T M C-C L)$ patches implanted after a lesion in the spinal cord was evaluated by assessing RhoA-GTP in tissue from the lesion site. The immunohistological labelling confirmed that in injured animals (non-treated) neuronal cells ( $\beta$ III tubulin positive cells) at the lesion site express activated RhoA (RhoA-GTP) (Fig. 4e). Quantitative assessment of RhoA (Fig. $4^{f}$-g) point to a decrease in RhoA-GTP when ibuprofen-loaded P(TMC-CL) scaffolds are implanted in comparison to injured animals (Fig. 4f). When comparing animals in which plain and ibuprofen-loaded P(TMC-CL) scaffolds were implanted both the results obtained by G-LISA (Fig. 4f) and Western blot (Fig. 4q) suggest that the drug promoted a decrease in RhoA-GTP.

In order to characterize the cellular populations present at the lesion site after implantation of the $\mathrm{P}(\mathrm{TMC}-\mathrm{CL})$ patches, tissue cryosections were prepared and labelled with specific cell markers. A large number of cells at the lesion site (delimited as depicted in Fig. 5 b) were found to be IBA-1 positive, indicating significant microglia/macrophage infiltration for all conditions tested (Fig. 5a). CD163, a marker for macrophages, is considered to label only perivascular microglia [28] and has also been used by several authors as marker for anti-inflammatory macrophages [29, 30, 31]. The histological analysis of the explanted tissue suggests increased levels of $\mathrm{CD}_{1} 6_{3}$ positive cells at the lesion site when ibuprofen-loaded $\mathrm{P}(\mathrm{TMC}-\mathrm{CL})$ patches are implanted. Similarly, $\beta$ III tubulin positive cells tend to be increased in these test groups. The infiltration at the lesion site of GFAP positive cells (a marker for astrocytic cell population) is also increased in animals that were implanted with $P(T M C-C L)$ scaffolds (Fig. 5a). 
In Fig. $5 \mathrm{C}$ representative images are presented of immunostainned cryosections collected at the lesion site of animals treated with ibuprofen-loaded $\mathrm{P}(\mathrm{TMC}-\mathrm{CL})$ patches. The cellular populations found in close proximity to the ibuprofen-loaded P(TMC-CL) scaffolds, near to the lesion epicentre, can be observed. Although the majority of the cells are IBA-1 positive cells, $\beta$ III tubulin and GFAP positive cells are also identified. Cellular infiltration in the fibrous layer of the scaffold was detected.

\title{
4 Discussion
}

It is commonly accepted that a multi-target strategy will be required to tackle the challenge of promoting nerve regeneration after $\mathrm{SCl}[1,17]$. Following the primary injury that interrupts axonal pathways, rewiring of the system is limited due to the lack of physical support, the activation of inhibitory pathways, and, ultimately, due to the formation of a glial scar that physically hinders axonal regeneration. In the present work, it is described the preparation of a bilayer patch easily implantable at the epidural space that combines physical support, guidance cues and can serve as reservoir for the in situ delivery of ibuprofen, a drug expected to reduce the levels of RhoA activation at the lesion site.

The use of aligned electrospun fibres to guide axonal growth has been previously explored in the literature both for the peripheral and the central nervous system regeneration (see [32] for a review). The first report using aligned electrospun fibres in vivo after $\mathrm{SCl}$ claimed a robust rostral regeneration, four weeks after implantation of poly(L-lactide) longitudinally aligned fibres. The success of the strategy was ascribed to the anisotropy of the substrates as modest regeneration was observed when randomly oriented fibres or solvent cast films were implanted [20]. Here, we report the successful preparation of $\mathrm{P}(\mathrm{TMC}-\mathrm{CL})$ preferentially aligned fibres. Fibre mean diameter was found to be in average $0650 \mu \mathrm{m}$, in accordance with that previously described for randomly oriented structures using the same solvent mixture [24] . The aligned fibres were collected onto solvent cast films in order to obtain a bilayer patch with improved mechanical properties. Nanofibrous scaffolds have, in general, weaker mechanical properties than bulky structures [33], particularly when constituted by aligned fibres [34]. This can compromise the success of an implantable device, as demonstrated in an early study showing that the folding of an electrospun fibrous fabric impaired nerve regeneration [35].

The vectorization through scaffolds of molecules that have a positive effect on regeneration holds great potential as a therapeutic strategy for $\mathrm{SCl}$ regeneration. In the present study, it is proposed the use of ibuprofen incorporated in a $\mathrm{P}(\mathrm{TMC}-\mathrm{CL})$ based patch, taking advantage of the reported inhibitory action of the drug on the RhoA pathway $[11,13]$. Bilayer ibuprofen-loaded patches were prepared by mixing the drug with the polymeric solution prior to electrospinning and solvent casting, for the preparation of the patch inner and outer layer, respectively. It was found that the bulk of ibuprofen is released from the $\mathrm{P}(\mathrm{TMC}-\mathrm{CL})$ bilayer scaffolds within the first $24 \mathrm{~h}$ of incubation. The observed ibuprofen release profile is therapeutically significant in the context of a $\mathrm{SCl}$, as the administration of the drug has been suggested to be particularly relevant in the sub-acute phase [13]. However, it is worthwhile mentioning that the release study was conducted under sink conditions, being media completely refreshed at each evaluation time point, which favours the complete wash out of the drug from the scaffold. Thus, it is expected that the drug release rate will be slower when the patches are implanted in the spinal cord due to diffusion constraints [36], extending the drug release period [13].

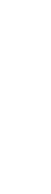


Our group has previously demonstrated the capacity of ibuprofen released from $\mathrm{P}(\mathrm{TMC}-\mathrm{CL})$ fibres to reduce prostaglandin $E_{2}$ secretion in human monocyte-derived macrophages [24]. Here, we tested the effect of ibuprofen loaded in P(TMC-CL) bilayer patches on the modulation of the RhoA pathway in a neuronal-like cell line. Although it was observed some variability in RhoA activity in different experiments, as described by others [13, 37], the obtained results point to a decrease on RhoA activation in cells pre-treated with the released drug, indicating ibuprofen bioactivity in vitro in this particular pathway.

As proof of concept for the application of ibuprofen-loaded patches in the context of a SCl, these materials were implanted in vivo in a dorsal hemisection rat SCI model. The study focused on the short-term response to the implantation of the patches, namely in terms of RhoA activation and cellular recruitment and infiltration. Previous studies demonstrating ibuprofen's role in the RhoA pathway in the context of $\mathrm{SCl}$ used the subcutaneous administration of the drug through osmotic pumps. The present study moves the concept a step forward with the implantation of a biodegradable [38] ibuprofen-loaded implantable device. It was demonstrated that P(TMC-CL) patches show appropriate mechanical properties to be implanted at the lesion site, wrapping the spinal cord tissue and allowing the fibrous layer to contact the injured tissue. The $P(T M C-C L)$ scaffolds under investigation were loaded with an ibuprofen dose of $500 \mu \mathrm{g} \mathrm{cm}^{-2}$, corresponding to approximately $0.75 \mathrm{mg} \cdot \mathrm{kg}^{-1}$. This drug dose is significantly lower than the $60 \mathrm{mg} \mathrm{kg}^{-1} \mathrm{day}^{-1}$ applied subcutaneously during 5 days $[\underline{11}, 13]$, but one should take into consideration that here the approach involves the delivery of the drug in situ. A previous study applying at the lesion site high doses of rolipram, a small molecule that enhances cAMP activity in neurons and favours nerve regeneration [39], reports deleterious effect on the animal survival (patch loaded with $65 \mathrm{\mu g} \mathrm{cm}^{-2}$ ) [40]. It is worth mentioning that the effective dose of rolipram is $1 \mathrm{mg} \cdot \mathrm{kg}^{-1}$ (subcutaneous administration) [41], 60 times less than that reported for ibuprofen [11, 13]. Ibuprofen loading applied in the present work took these data into consideration and the results demonstrate that the implantation of ibuprofenloaded $\mathrm{P}(\mathrm{TMC}-\mathrm{CL})$ scaffolds did not compromise animal survival rate. Moreover, as hypothesised, tissue from animals implanted with ibuprofen-loaded $P(T M C-C L)$ scaffolds expressed lower levels of RhoA-GTP in comparison to non-treated animals, indicating that an effective dose of ibuprofen has been released from the patch in the 5 -day implantation period. In this study, some variability is observed that may be raised by the inherent variation associated with the experimental injury model. Indeed, dorsal hemisection can involve variation on lesion extension, interfering in the results obtained onwards [12] .

Taking into consideration that cell infiltration and recruitment to the lesion site are determinant in tissue response to injury [42] and to complement the biofunctionality characterization of the developed patches the cell populations at the lesion site after implantation of $\mathrm{P}(\mathrm{TMC}-\mathrm{CL})$ scaffolds were evaluated. Independently of the applied treatment, the semi-quantitative analysis of the cells present at the lesion site show that IBA-1+ cells are the most abundant cell type. Although a previous report showed a reduction on microgliosis after 3 days of treatment with ibuprofen administrated subcutaneously [14] , here no evident difference in IBA-1+ cell presence was identified. It has been noticed, however, that implantation of $\mathrm{P}(\mathrm{TMC}-\mathrm{CL})$ scaffolds, in particular when loaded with ibuprofen, can favour $\mathrm{CD}_{1} 6_{3}{ }^{+}$cell recruitment. $\mathrm{CD}_{1} 6_{3}$ is a marker for macrophages and it has been mainly associated with an anti-inflammatory phenotype in human macrophages $[29,30,43]$ as well as in rat derived macrophages [31], suggesting that ibuprofen released from $\mathrm{P}(\mathrm{TMC}-\mathrm{CL})$ scaffolds can contribute to a positive resolution of the inflammatory process after $\mathrm{SCl}$. Moreover, despite being a short-term study, our data points to an increase of the number of $\beta$ III-tubulin and GFAP positive cells

INSTITUTO DE INVESTİGAÇÃO E INOVACCÃO EM SAÚDE UNIVERSIDADE DO PORTO

Rua Alfredo Allen, 208 4200-135 Porto Portugal +351220408800 
at the lesion site when $\mathrm{P}(\mathrm{TMC}-\mathrm{CL})$ patches are implanted. Although astrogliosis has been traditionally considered as a hallmark for CNS pathology [44], astrocytes have shown to play a critical role in the process of axonal regeneration after $\mathrm{SCl}$ [45], being in the frontline of neuronal migration [20]. Ibuprofen-loaded scaffolds showed also to support axonal growth ( $\beta$ III-tubulin cells) in the lesion periphery and cellular infiltration.

Altogether, the presented data put forward ibuprofen-loaded P(TMC-CL) scaffolds as a novel multifactorial approach to target the inhibitory milieu at $\mathrm{SCl}$ site. The long-term implantation of these scaffolds will unveil whether the reported reduction on RhoA-GTP levels and the alterations on the cellular populations at the lesion site can prompt functional regeneration in the aftermath of $\mathrm{SCl}$.

\section{Conclusions}

This study shows the successful preparation of bilayer $\mathrm{P}(\mathrm{TMC}-\mathrm{CL})$ scaffolds comprising longitudinally aligned $\mathrm{P}(\mathrm{TMC}-\mathrm{CL})$ fibres loaded with ibuprofen. It is demonstrated that ibuprofen-loaded patches reduce RhoA activation in vitro in ND7/23 cells and in vivo when implanted in a rat dorsal hemisection $\mathrm{SCl}$ model. These structures supported neuronal cell growth and favoured cellular infiltration putting forward ibuprofen-loaded $\mathrm{P}(\mathrm{TMC}-\mathrm{CL}$ ) patches as a new solution to tame the inhibitory environment at the $\mathrm{SCl}$ site. Furthermore, the presented $3 \mathrm{D}$ structures may serve as depot of different signals towards engineering a multi-functional device to address $\mathrm{SCI}$.

\section{Acknowledgements}

This work was financed by FEDER funds through the Programa Operacional Factores de Competitividade - COMPETE and by Portuguese funds through FCT - Fundação para a Ciência e a Tecnologia in the framework of the project PEst-C/SAU/LAooo2/2011 and PTDC/CTMNAN/115124/2009. LR Pires, CDF Lopes and DN Rocha thank FCT for their PhD grants (SFRH/BD/46015/2008, SFRH/BD/77933/2011 and SFRH/BD/64079/2009). The authors wish to thank Mónica M Sousa, Ana Marques and Marlene Morgado (Nerve Regeneration group, IBMCli3S) for the help in animal experimentation and Cecília Alves and Daniela Sousa (INEB|i3S) for assistance in image analysis. Authors acknowledge the Centro de Materiais da Universidade do Porto (CEMUP; REEQ/1062/CTM/2005 from FCT) for SEM and ${ }^{1 H}$ NMR analysis and Dr. Sérgio Simões for the possibility to use the HPLC equipment at Bluepharma (Coimbra).

\section{Compliance with ethical standards}

\section{Conflict of interests}

The authors declares that they have no competing interests.

\section{西}



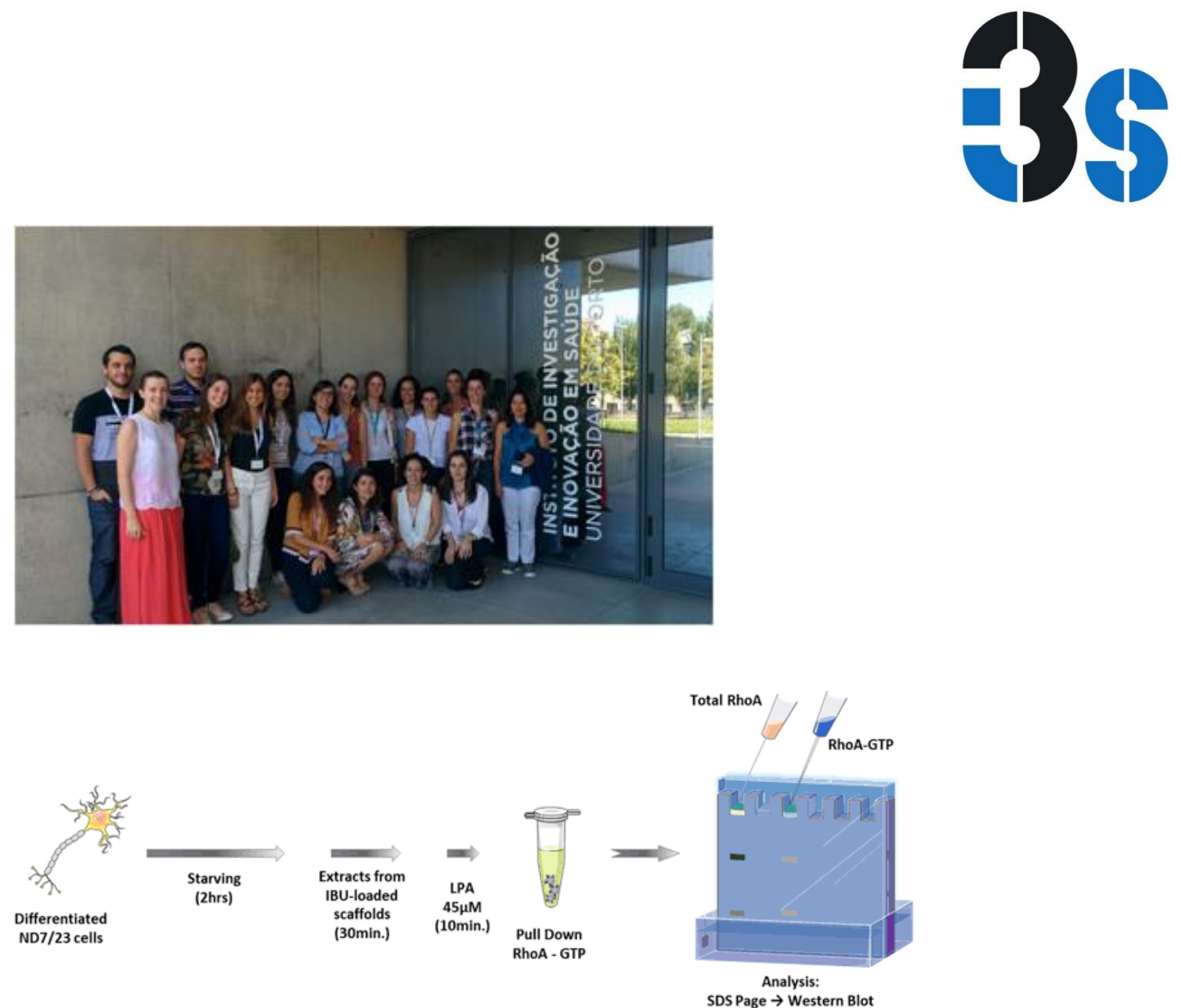

Fig. 1 Schematic representation of the experimental setup for RhoA determination in vitro. Differentiated (200 nM NGF, 1 mM CAMP, 2 days) ND7/23 cells were starved (DMEM without serum) for $2 \mathrm{~h}$ and incubated for $30 \mathrm{~min}$ with extracts from ibuprofen loaded scaffolds. Ibuprofen solution $(500 \mu \mathrm{M})$ was used as control. Subsequently, cells were activated with LPA for $10 \mathrm{~min}$. RhoA-GTP was pulled down using Rhotekin RBD-coupled beads and quantified by Western blot 

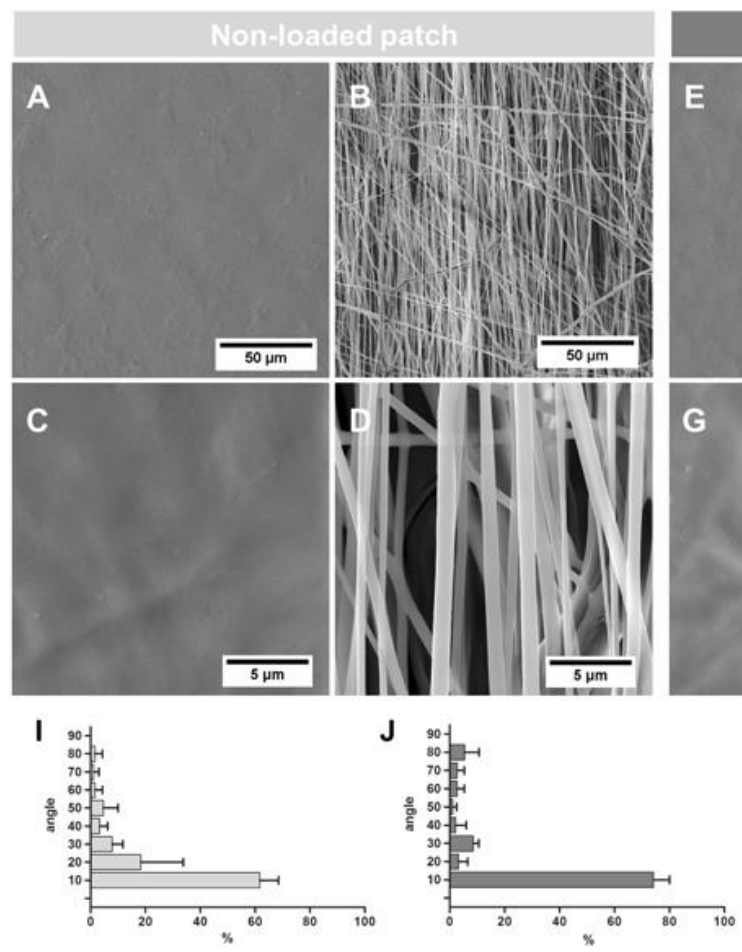

Fig. 2 Characterization of the $P(T M C-C L)$ patch bilayers. Scanning electron microscopy (SEM) photomicrographs of a-d non-loaded and e-h ibuprofen-loaded $\mathrm{P}(\mathrm{TMC}-\mathrm{CL})$ patches. Images a, $\mathbf{c}$, e and $\mathbf{g}$ show the patch outer layer composed by solvent cast films; images $\mathbf{b}, \mathbf{d}$, $\mathbf{f}$ and $\mathbf{h}$ show the inner layer made of preferentially aligned fibres prepared by electrospinning. $\mathbf{i}$ and $\mathbf{j}$ Graphic representation of fibre alignment quantification for $\mathbf{i}$ non-loaded and $\mathbf{j}$ ibuprofen-loaded fibres. Bars represent average and error bars show standard deviation for measurements performed in three independent samples $(n=3)$. k Fibre mean diameter \pm standard deviation as calculated from image analysis of three independent samples ( $n=3$ samples; 100 measurements per sample)

A

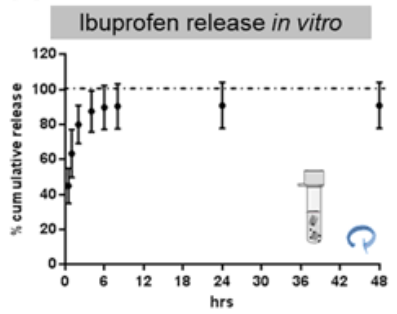

B

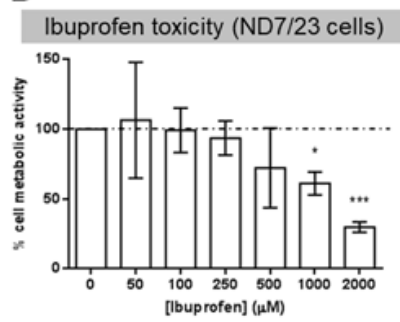

C

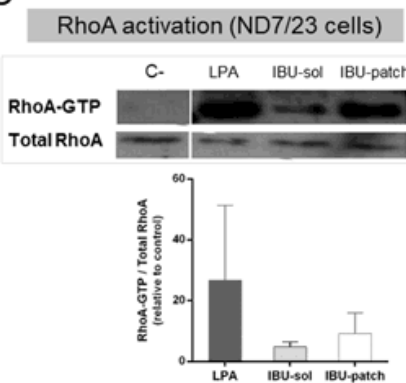

Fig. 3 Ibuprofen release and bioactivity in vitro. a Cumulative release of ibuprofen from $P(T M C-C L)$ patches $(n=7)$ when incubated in $\mathrm{PBS}\left(37^{\circ} \mathrm{C}\right)$, as determined by high-pressure liquid chromatography (HPLC). b ND7/23 cell viability after $24 \mathrm{~h}$ in contact with ibuprofen (in solution) at increasing concentrations $(n=5)$. Bars represent average value and error bars show standard deviation. Represents $* p<0.05$ and $* * * p<0.001$. c RhoA activation in ND7/23 cells as determined by Western blot after precipitation of the active form of RhoA (RhoA-GTP). RhoA-GTP/total RhoA was calculated

INSTITUTO DE INVESTİGAÇÃO E INOVAÇÃO EM SAÚDE UNIVERSIDADE DO PORTO

Rua Alfredo Allen, 208 4200-135 Porto Portugal +351 220408800 
relative to RhoA basal activation ( $\left.C^{-}\right)(n=3)$. Stimulation with LPA ( $45 \mu \mathrm{M}, 10 \mathrm{~min}$ ) and pre-treatment with ibuprofen (IBU-sol) or ibuprofen released from $\mathrm{P}(\mathrm{TMC}-\mathrm{CL}$ ) patches (IBU-patch) were tested

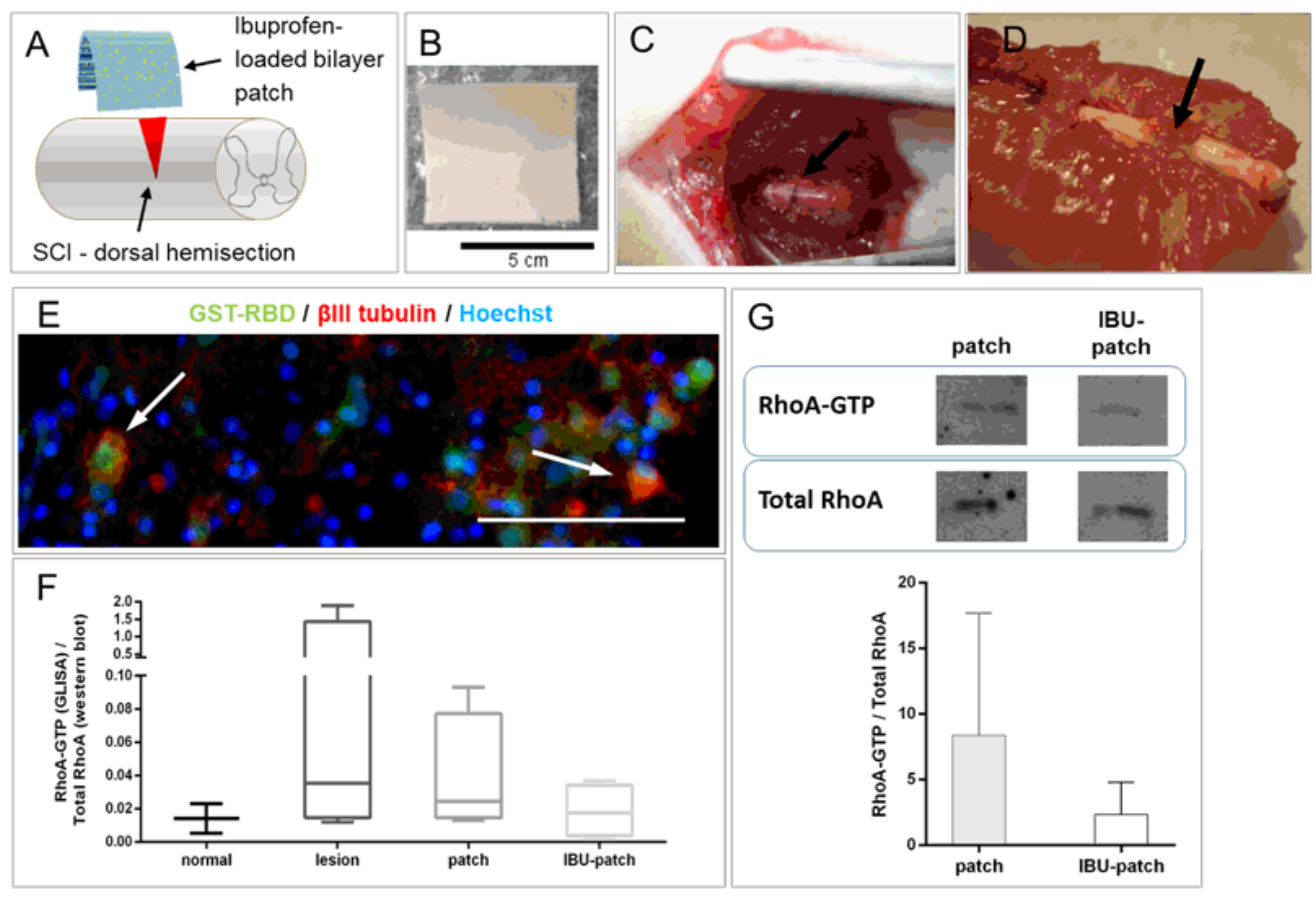

Fig. 4 Implantation of P(TMC-CL) patches and RhoA-GTP detection in vivo. a Illustration representing the experimental setup. Photographs show the $\mathrm{P}(\mathrm{TMC}-\mathrm{CL})$ bilayer patch $\mathbf{b}$ prior implantation (gamma sterilized); c after implantation in an injured (dorsal hemisection) spinal cord; and $\mathbf{d}$ as collected after animal euthanasia ( 5 days post implantation). Arrows indicate the place of the injury and the P(TMC-CL) patch. e In situ pull down of RhoA-GTP and immunodetection of GST-RBD (that binds to RhoA-GTP; shown in green) and BIII tubulin (shown in red) in cryosections of spinal cord tissue of non-treated animals. Double-labelled cells are indicated by white arrows. Scale bar: $100 \mu \mathrm{m}$. f RhoA-GTP detected in tissue as determined by GLISA. The presented values were normalized by the total RhoA detected by Western blot ( $n=4$, except for normal tissue where $n=2)$. g RhoA activation levels detected by Western blot after pull down of the activated form RhoA-GTP $(n=3)$ (color figure online) 


\begin{tabular}{|c|c|c|c|}
\hline & & & \\
& lesion & patch & IBU-patch \\
\hline IBA-1 & +++ & ++ & +++ \\
\hline CD163 & + & + & ++ \\
\hline GFAP & + & ++ & ++ \\
\hline BIII-tubulin & + & ++ & ++ \\
\hline
\end{tabular}
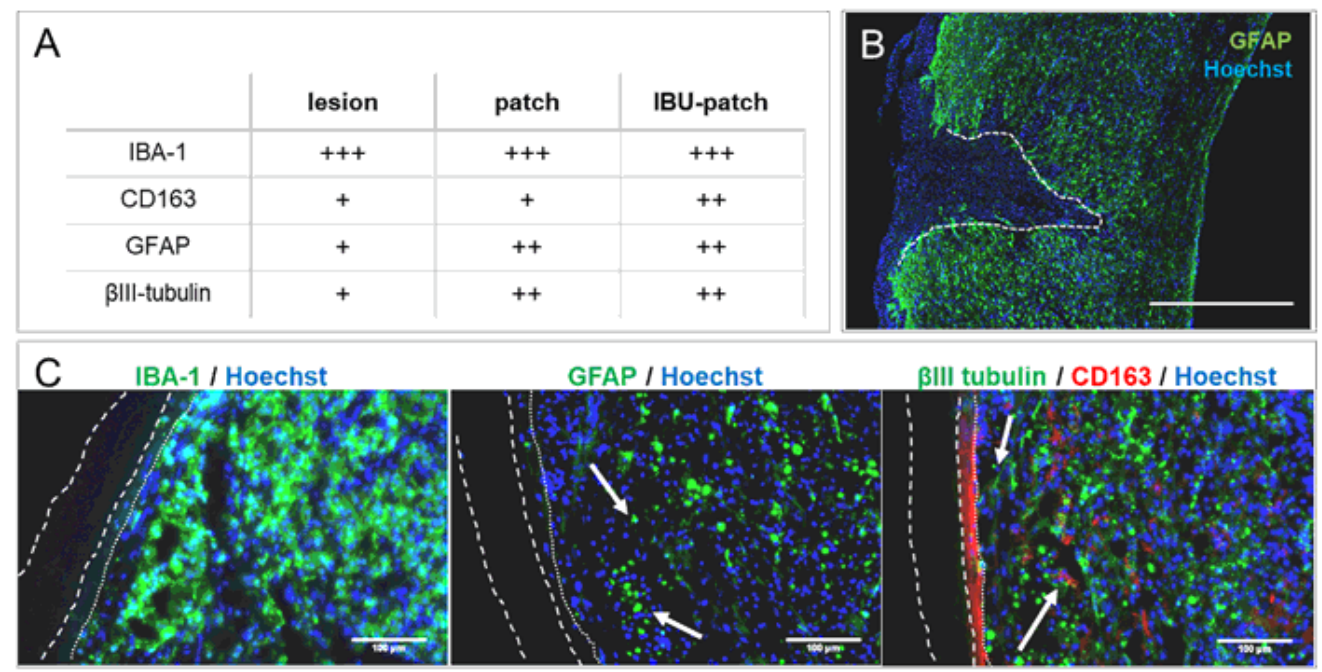

Fig. 5 Immunohistochemical analysis of tissues. a Semi-quantitative analysis of the immunohistochemistry images. Scoring system: $(+/-)$ rare cells; $(+)$, few cells; $(++)$, some cells; $(+++)$, many cells. $\mathbf{b}$ Fluorescence microscopy image showing the delimitation of the lesion site, 5 days after dorsal hemisection (scale bar: $1000 \mu \mathrm{m}$ ). c Detail of cells found close to the ibuprofen-loaded patch 5 days after lesion. The site of the bilayer scaffold is delimited with the white dashed lines. GFAP, $\beta$ III tubulin and IBA-1 positive labelling are shown in green (left, centre and right panels, respectively) and $\mathrm{CD}_{16} 6$ appears in red (right panel). Cell nuclei are labelled by Hoechst and shown in blue. Scale bar: $100 \mu \mathrm{m}$ (color figure online) 


\section{REFERENCES}

1.Pires $L R$, Pêgo AP. Bridging the lesion-engineering a permissive substrate for nerve regeneration. Regen Biomater. 2015;2(3):203-14.

2 .Ramer LM, Ramer MS, Bradbury EJ. Restoring function after spinal cord injury: towards clinical translation of experimental strategies. Lancet Neurol. 2014;13(12):1241-56.

3.Schwab JM, Brechtel K, Mueller CA, Failli V, Kaps HP, Tuli SK, et al. Experimental strategies to promote spinal cord regeneration - An integrative perspective. Prog Neurobiol. 2006;78(2):91-116.

4.Dergham P, Ellezam B, Essagian C, Avedissian H, Lubell WD, McKerracher L. Rho signaling pathway targeted to promote spinal cord repair. J Neurosci. 2002;22(15):6570-7.

5 .McKerracher L, Higuchi H. Targeting Rho to stimulate repair after spinal cord injury. J Neurotrauma. 2006;23(34):309-17.

6.Monnier PP, Sierra A, Schwab JM, Henke-Fahle S, Mueller BK. The Rho/ROCK pathway mediates neurite growth-inhibitory activity associated with the chondroitin sulfate proteoglycans of the CNS glial scar. Mol Cell Neurosci. 2003;22(3):319-30.

7 .Rossignol S, Schwab M, Schwartz M, Fehlings MG. Spinal cord injury: Time to move? J Neurosci. 2007;27(44):11782-92.

8.Zhou Y, Su Y, Li BL, Liu F, Ryder JW, Wu X, et al. Nonsteroidal anti-inflammatory drugs can lower amyloidogenic $A$ beta(42) by inhibiting Rho. Science. 2003;302(5648):1215-7.

9.Mitchell JA, Akarasereenont P, Thiemermann C, Flower RJ, Vane JR. Selectivity of nonsteroidal antiinflammatory drugs as inhibitors of constitutive and inducible cyclooxygenase. Proc Natl Acad Sci USA. 1993;90(24):11693-7.

10. Rainsford KD. Ibuprofen: pharmacology, efficacy and safety. Inflammopharmacology. 2009;17(6):275-342.

11 .Fu Q, Hue J, Li S. Nonsteroidal anti-inflammatory drugs promote axon regeneration via RhoA inhibition. J Neurosci. 2007;27(15):4154-64.

12 .Sharp KG, Yee KM, Stiles TL, Aguilar RM, Steward O. A re-assessment of the effects of treatment with a nonsteroidal anti-inflammatory (ibuprofen) on promoting axon regeneration via RhoA inhibition after spinal cord injury. Exp Neurol. 2013;248:321-7.

13.Wang X, Budel S, Baughman K, Gould G, Song KH, Strittmatter SM. Ibuprofen enhances recovery from spinal cord injury by limiting tissue loss and stimulating axonal growth. J Neurotrauma. 2009;26(1):81-95.

14. Redondo-Castro E, Navarro X. Chronic ibuprofen administration reduces neuropathic pain but does not exert neuroprotection after spinal cord injury in adult rats. Exp Neurol. 2014;252:95-103.

15.Xing B, Li H, Wang H, Mukhopadhyay D, Fisher D, Gilpin CJ, et al. RhoA-inhibiting NSAIDs promote axonal myelination after spinal cord injury. Exp Neurol. 2011;231(2):247-60.

16.Kopp MA, Liebscher T, Niedeggen A, Laufer S, Brommer B, Jungehulsing GJ, et al. Small-molecule-induced Rho-inhibition: NSAIDs after spinal cord injury. Cell Tissue Res. 2012;349(1):119-32.

17 .McCreedy DA, Sakiyama-Elbert SE. Combination therapies in the CNS: engineering the environment. Neurosci Lett. 2012;519(2):115-21.

18.Corey JM, Lin DY, Mycek KB, Chen Q, Samuel S, Feldman EL, et al. Aligned electrospun nanofibers specify the direction of dorsal root ganglia neurite growth. J Biomed Mater Res A. 2007;83(3):636-45.

19. Yao L, O'Brien N, Windebank A, Pandit A. Orienting neurite growth in electrospun fibrous neural conduits. J Biomed Mater Res B Appl Biomater. 2009;90(2):483-91. 
20. Hurtado A, Cregg JM, Wang HB, Wendell DF, Oudega M, Gilbert RJ, et al. Robust CNS regeneration after complete spinal cord transection using aligned poly-I-lactic acid microfibers. Biomaterials. 2011;32(26):6068-79.

21.Pêgo AP, Poot AA, Grijpma DW, Feijen J. Biodegradable elastomeric scaffolds for soft tissue engineering. J Control Release. 2003;87(1-3):69-79.

22. Vleggeert-Lankamp CLAM, Wolfs J, Pêgo AP, Van Den Berg R, Feirabend H, Lakke E. Effect of nerve graft porosity on the refractory period of regenerating nerve fibers: laboratory investigation. J Neurosurg. 2008;109(2):294-305.

23 . Rocha DN, Brites P, Fonseca C, Pêgo AP. Poly(trimethylene carbonate-co- $\varepsilon$-caprolactone) promotes axonal growth. PLoS ONE. 2014;9(2):e88593.

24 .Pires LR, Guarino V, Oliveira MJ, Ribeiro CC, Barbosa MA, Ambrosio L, et al. Ibuprofen-loaded poly(trimethylene carbonate-co- $\varepsilon$-caprolactone) electrospun fibers for nerve regeneration. J Tissue Eng Regen Med. 2016;10(3):E154.

25 .Pires LR, Rocha DN, Ambrosio L, Pêgo AP. The role of the surface on microglia function: implications for central nervous system tissue engineering. J R Soc Interface. 2015;12:103.

26.Pêgo AP, Poot AA, Grijpma DW, Feijen J. Copolymers of trimethylene carbonate and epsilon-caprolactone for porous nerve guides: Synthesis and properties. J Biomater Sci Polym Ed. 2001;12(1):35-53.

27.Pêgo AP, Vleggeert-Lankamp CLAM, Deenen M, Lakke EAJF, Grijpma DW, Poot AA, et al. Adhesion and growth of human schwann cells on trimethylene carbonate (co)polymers. J Biomed Mater Res A. 2003;67(3):87685 .

28.Polfliet MMJ, Fabriek BO, Daniëls WP, Dijkstra CD, van den Berg TK. The rat macrophage scavenger receptor CD163: expression, regulation and role in inflammatory mediator production. Immunobiology. 2006;211(6-8):41925 .

29. Gordon S. Alternative activation of macrophages. Nat Rev Immunol. 2003;3(1):23-35.

30.Durafourt BA, Moore CS, Zammit DA, Johnson TA, Zaguia F, Guiot MC, et al. Comparison of polarization properties of human adult microglia and blood-derived macrophages. Glia. 2012;6o(5):717-27.

31.Ma SF, Chen YJ, Zhang JX, Shen L, Wang R, Zhou JS, et al. Adoptive transfer of M2 macrophages promotes locomotor recovery in adult rats after spinal cord injury. Brain Behav Immun. 2015;45:157-70.

32.Xie J, MacEwan MR, Schwartz AG, Xia Y. Electrospun nanofibers for neural tissue engineering. Nanoscale. 2010;2(1):35-44.

33.Teo WE, Ramakrishna S. A review on electrospinning design and nanofibre assemblies. Nanotechnology. $2006 ; 17(14)$ :R89-R106.

34.Zhu Y, Wang A, Shen W, Patel S, Zhang R, Young WL, et al. Nanofibrous patches for spinal cord regeneration. Adv Funct Mater. 2010;20(9):1433-40.

35 . Meiners S, Ahmed I, Ponery AS, Amor N, Harris SL, Ayres V, et al. Engineering electrospun nanofibrillar surfaces for spinal cord repair: A discussion. Polym Int. 2007;56(11):1340-8.

36 .Zhong Y, Bellamkonda RV. Biomaterials for the central nervous system. J R Soc Interface. 2008;5(26):957-75

37. Dill J, Patel AR, Yang XL, Bachoo R, Powell CM, Li S. A molecular mechanism for ibuprofen-mediated RhoA inhibition in neurons. J Neurosci. 2010;30(3):963-72.

38 .Pêgo AP, Van Luyn MJA, Brouwer LA, Van Wachem PB, Poot AA, Grijpma DW, et al. In vivo behavior of poly(1,3-trimethylene carbonate) and copolymers of 1,3-trimethylene carbonate with $D, L$-lactide or epsiloncaprolactone: Degradation and tissue response. J Biomed Mater Res A. 2003;67(3):1044-54.

INSTITUTO DE INVESTİGAÇÃO EINOVAÇÃO UNIVERSIDADE DO PORTO

Rua Alfredo Allen, 208 4200-135 Porto Portugal +351220 408800 
39 .Pearse DD, Pereira FC, Marcillo AE, Bates ML, Berrocal YA, Filbin MT, et al. cAMP and Schwann cells promote axonal growth and functional recovery after spinal cord injury. Nat Med. 2004;10(6):610-6.

40 .Downing TL, Wang A, Yan ZQ, Nout Y, Lee AL, Beattie MS, et al. Drug-eluting microfibrous patches for the local delivery of rolipram in spinal cord repair. J Control Release. 2012;161(3):910-7.

41.Schaal SM, Garg MS, Ghosh M, Lovera L, Lopez M, Patel M et al. The therapeutic profile of rolipram, PDE target and mechanism of action as a neuroprotectant following spinal cord injury. PLoS ONE. 2012;7(9): e43634.

42 .Sakiyama-Elbert S, Johnson PJ, Hodgetts SI, Plant GW, Harvey AR. Scaffolds to promote spinal cord regeneration. Handb Clin Neurol. 2012;109:575-94.

43 .Fuentes-Duculan J, Suárez-Farĩas M, Zaba LC, Nograles KE, Pierson KC, Mitsui H, et al. A subpopulation of CD163-positive macrophages is classically activated in psoriasis. J Invest Dermatol. 2010;130(10):2412-22.

44 .Karimi-Abdolrezaee S, Billakanti R. Reactive astrogliosis after spinal cord injury-beneficial and detrimental effects. Mol Neurobiol. 2012;46(2):251-64.

45 .Chu T, Zhoul H, Li F, Wang T, Lu L, Feng S. Astrocyte transplantation for spinal cord injury: current status and perspective. Brain Res Bull. 2014;107:18-30. 